\title{
BANACH-HAUSDORFF LIMITS
}

\author{
W. F. EBERLEIN
}

1. Introduction. Let $m$ denote the Banach lattice of bounded real sequences $x=\left(x_{0}, x_{1}, x_{2}, \cdots\right)$ with $\|x\|=\sup _{n}\left|x_{n}\right|$ and $x \geqq y$ if $x_{n} \geqq y_{n}$ for all $n$. Let $S$ denote the shift operator $S\left(x_{0}, x_{1}, x_{2}, \cdots\right)$ $=\left(x_{1}, x_{2}, \cdots\right)$. Banach ${ }^{1}$ has established the existence of real-valued functionals $L(x)$ defined over $m$ with the properties

(I) $L(a x+b y)=a L(x)+b L(y)(a, b$ real $)$;

(II) $L(1)=1$;

(III) $L(x) \geqq 0$ if $x \geqq 0$;

(IV) $L(S x)=L(x)$.

The conditions (I)-(IV) imply that

$$
L_{*}(x)=\lim _{n} \inf x_{n} \leqq L(x) \leqq \lim _{n} \sup x_{n}=L^{*}(x),
$$

whence a Banach limit of a convergent sequence is the ordinary limit.

Designating the class of regular Hausdorff ${ }^{2}$ transformations by $\mathfrak{S}$, we term $L(x)$ a Banach-Hausdorff $(B-H)$ functional or limit if in addition to (I)-(IV) it satisfies

(V) $L(H x)=L(x)(B \in \mathfrak{W})$.

The corresponding regularity property of B-H limits then takes the form: If the bounded sequence $x$ is summable to $a$ by some regular Hausdorff method, then $L(x)=a$ for every B-H functional $L$.

The obvious problem is the existence of B-H limits. Our solution yields simultaneously the existence and the domain of uniquenessthat is, the set of $x$ in $m$ on which all B-H functionals coincide. ${ }^{3}$

2. Hausdorff lore. We recall the following properties of the class $\mathfrak{W}$ of regular Hausdorff transformations: $\mathfrak{W}$ is the convex Abelian semi-group of linear transformations of $m$ into itself defined by the Toeplitz matrices $\left(a_{m n}\right)$, where

$$
\begin{aligned}
a_{m n} & =C_{m, n} \int_{0}^{1} u^{n}(1-u)^{m-n} d \alpha(u) & & (n \leqq m) \\
& =0 & & (n>m) ;
\end{aligned}
$$

\footnotetext{
Presented to the Society, September 2, 1949; received by the editors August 1, 1949.

' S. Banach, Theorie des operations lineaires, Warsaw, 1932.

2 F. Hausdorff, Math. Zeit, vol. 9 (1921) pp. 74-109, 280-299.

3 The domain of uniqueness of the ordinary Banach limits has been determined by G. G. Lorentz, Acta Math. vol. 80 (1948) pp. 167-190.
} 
and the $\alpha(u)$ are real functions of bounded variation (BV) in the interval $(0,1)$ satisfying the end conditions: $\alpha(0)=\alpha(0+)=0$, $\alpha(1)=1$.

$\mathrm{H}$ in $\mathfrak{Q}$ is termed definite or completely regular ${ }^{4}$ (c.r.) if the generating function $\alpha(u)$ is non-decreasing or, equivalently, if all the matrix elements $a_{m n}$ are non-negative. If $H$ is c.r., $\|H\|=1$ and

$$
L_{*}(x) \leqq L_{*}(H x) \leqq L^{*}(H x) \leqq L^{*}(x) .
$$

The standard decomposition of BV functions yields the canonical resolution of a regular $H$ : .

$$
H=a H_{1}-b H_{2} \quad\left(H_{1}, H_{2} \text { c.r.; } a-b=1, b \geqq 0\right) .
$$

Henceforth we need consider only the convex semi-group $\mathfrak{S}_{+} \subset \mathfrak{C}$ of c.r. Hausdorff transformations and may replace (V) with the equivalent condition

(V bis) $L(H x)=L(x)\left(H \in \mathfrak{S}_{+}\right)$.

The following conventions will materially shorten our work:

Definition 1. $C$ is the class of convergent sequences; $C_{0}$ the subclass of sequences converging to 0 (null sequences). $x \sim y$ if and only if $x-y$ $\in C_{0}$.

Clearly $\sim$ is a congruence relation under addition, scalar multiplication, and the operations $H$.

Definition 2. $P_{+}(x)=\inf _{H \in \mathfrak{S}_{+}} L^{*}(H x) ; \quad P_{-}(x)=-P_{+}(-x)$ $=\sup _{H \in \mathfrak{S}_{+}} L_{*}(H x)$.

Our basic results may now be formulated as

TheOREM 1. (a) A n.a.s.c. that the linear functional $L(x)$ on $m$ be a $B-H$ functional is that

$$
P_{-}(x) \leqq L(x) \leqq P_{+}(x) \quad(x \in m) .
$$

(b) $B$-H functionals exist.

(c) $A$ n.a.s.c. that all $B-H$ functionals coincide at $x$ is that $P_{-}(x)$ $=P_{+}(x)$.

That B-H functionals must satisfy the inequality (5) is an immediate consequence of (1) and (V bis). The condition $P_{-}(x)=P_{+}(x)$ is then obviously sufficient to ensure coincidence at $x$.

3. Convexity. To establish the remaining assertions of Theorem 1 , we derive the convexity and other necessary properties of $P_{+}(x)$, and then adapt the Hahn-Banach ${ }^{1}$ extension procedure. The decisive role of the Hölder-Cesàro transformation $H_{0} x=y$, where $y_{n}=(n+1)^{-1}$

4 Cf. G. H. Hardy, Divergent series, Oxford, 1949. 
$\cdot\left(x_{0}+\cdots+x_{n}\right)$ corresponds to $\alpha(u)=u$, is due to the following elementary property:

LEMma 1. $H_{0} S x \sim H_{0} x \sim S H_{0} x$.

The requisite properties of $P_{ \pm}(x)$ are

Lemma 2. (a) $P_{+}(x+y) \leqq P_{+}(x)+P_{+}(y)$;

(b) $P_{+}(a x)=a P_{+}(x)(a \geqq 0)$;

(c) $P_{-}(x) \leqq P_{+}(x)$;

(d) $\left|P_{ \pm}(x)\right| \leqq\|x\| ; P_{ \pm}(x) \geqq 0$ if $x \geqq 0$;

(e) If $x \in C, P_{ \pm}(x)=\lim _{n} x_{n}$ and $P_{ \pm}(x+y)=\lim _{n} x_{n}+P_{ \pm}(y)$; in particular, $P_{ \pm}(x)=P_{ \pm}(y)$ if $x \sim y$;

(f) $P_{ \pm}(H x)=P_{ \pm}(x)\left(H \in \mathfrak{S}_{+}\right)$;

(g) $P_{ \pm}(S x)=P_{ \pm}(x)$

(h) $P_{ \pm}(S x-x)=0$;

(i) $P_{ \pm}(H x-x)=0\left(H \in \mathfrak{Q}_{+}\right)$.

Proof. (a) Given $\epsilon>0$, there exist $H_{1}, H_{2}$ in $\mathfrak{W}_{+}$such that $L^{*}\left(H_{1} x\right)$ $\leqq P_{+}(x)+\epsilon, L^{*}\left(H_{2} y\right) \leqq P_{+}(y)+\epsilon$. Set $H_{3}=H_{2} H_{1}=H_{1} H_{2}$. Then $L^{*}\left(H_{3} x\right)$ $=L^{*}\left(H_{2} H_{1} x\right) \leqq L^{*}\left(H_{1} x\right) \leqq P_{+}(x)+\epsilon$. Similarly $L^{*}\left(H_{3} y\right) \leqq P_{+}(y)+\epsilon$. Hence $P_{+}(x+y) \leqq L^{*}\left(H_{3}(x+y)\right) \leqq L^{*}\left(H_{3} x\right)+L^{*}\left(H_{3} y\right) \leqq P_{+}(x)+P_{+}(y)$ $+2 \epsilon$. Since $\epsilon>0$ was arbitrary, $P_{+}(x+y) \leqq P_{+}(x)+P_{+}(y)$.

(b), (d), and (e) are obvious, and (c) follows on setting $y=-x$ in (a). (f) is an easy consequence of the definition of $P_{ \pm}(x)$ by way of (3) and the semi-group property of $\mathfrak{L}_{+}$. Lemma 1 and (e), (f) now yield

(g) $P_{ \pm}(S x)=P_{ \pm}\left(H_{0} S x\right)=P_{ \pm}\left(H_{0} x\right)=P_{ \pm}(x)$;

(h) $P_{ \pm}(S x-x)=P_{ \pm}\left(H_{0} S x-H_{0} x\right)=0$.

To establish (i) note the ergodic ${ }^{5}$ property $\left\|H_{n} H-H_{n}\right\| \leqq 2(n+1)^{-1}$ of the operators $H_{n}=(n+1)^{-1} \sum_{0}^{n} H^{i} \in \mathfrak{S}_{+}$. Thus $\left|P_{ \pm}(H x-x)\right|$ $=\left|P_{ \pm}\left(\left(H_{n} H-H_{n}\right) x\right)\right| \leqq\left\|\left(H_{n} H-H_{n}\right) x\right\| \leqq 2(n+1)^{-1}\|x\| . \quad n$ being an arbitrary integer, $P_{ \pm}(H x-x)=0$.

We complete the proof of Theorem 1 . That any linear $L$ satisfying (5) is a B-H functional is now immediate: (II) follows from the identity $P_{ \pm}(1)=1$; (III) from Lemma 2(d); the identities $L(S x-x)$ $=0$ (IV) and $L(H x-x)=0$ (V bis) from Lemma 2 , (h) and (i) respectively.

Application of the Hahn-Banach extension theorem to the convex function $P_{+}(x)$ yields linear functionals $L$ such that $L(x) \leqq P_{+}(x)$. But then $L(x)=-L(-x) \geqq-P_{+}(-x)=P_{-}(x)$, whence the $L$ satisfy (5).

${ }^{5}$ Cf. W. F. Eberlein, Proc. Nat. Acad. Sci. U.S.A. vol. 34 (1948) pp. 43-47; Trans. Amer. Math. Soc. vol. 67 (1949) pp. 217-240. 
Finally, the necessity of the condition $P_{-}(x)=P_{+}(x)$ for coincidence of all B-H functionals at $x$ is implicit in the extension procedure: If $P_{-}(y)<P_{+}(y)$ for some $y$ in $m$, the value of $L(y)$ can be chosen arbitrarily in the interval $P_{-}(y) \leqq L(y) \leqq P_{+}(y)$. For let $L(x)$ $=\lim _{n} x_{n}(x \in C)$, and recall that we may continue $L$ into the subspace $x+a y(x \in C, a$ real) by choosing $L(y)$ arbitrarily in the interval

$$
\sup _{x \in c}\left\{P_{-}(x+y)-L(x)\right\} \leqq L(y) \leqq \inf _{x \in c}\left\{P_{+}(x+y)-L(x)\right\} .
$$

But the second inequality reduces to the first (Lemma 2(e)), and our assertion follows on continuing $L$ into the whole space $m$.

Further developments will appear in a second note.

UNIVERSITY OF WISCONSIN 